\title{
Erosion Remineralization Efficacy of Gel-to-Foam Fluoride Toothpastes in situ: A Randomized Clinical Trial
}

\author{
Marc Nehme $^{\mathrm{a}}$ Peter Jeffery ${ }^{\mathrm{a}}$ Stephen Mason ${ }^{\mathrm{a}}$ Frank Lippert ${ }^{\mathrm{b}}$ \\ Domenick T. Zero ${ }^{b}$ Anderson T. Hara ${ }^{b}$ \\ ${ }^{a}$ GSK Consumer Healthcare R\&D, Weybridge, UK; ${ }^{b}$ Oral Health Research Institute, Indiana University School of Dentistry, \\ Indianapolis, Ind., USA
}

\section{Key Words}

Dental erosion · Fluoride · Gel-to-foam toothpaste · In situ model $\cdot$ Surface microhardness

\begin{abstract}
This single-center, randomized, placebo-controlled, fourtreatment, four-period crossover study compared the enamel remineralization effects of low- and medium-abrasivity gel-to-foam toothpastes and a reference toothpaste (all $1,450 \mathrm{ppm}$ fluoride as $\mathrm{NaF}$ ) versus placebo toothpaste ( $0 \mathrm{ppm}$ fluoride) using a short-term in situ erosion model. Subjects $(n=56)$ wearing a palatal appliance holding acidsoftened bovine enamel specimens brushed their teeth with the test toothpastes. Thereafter, the specimens were removed for analysis of percent surface microhardness recovery (\%SMHR) and percent relative erosion resistance (\%RER) at 2,4 , and $8 \mathrm{~h}$. Both low- and medium-abrasivity gel-to-foam fluoride toothpastes and the reference toothpaste provided significantly greater \%SMHR than placebo at all assessment time points (all $p<0.05$ ). No statistically significant difference of \%SMHR was observed between the fluoride treatment groups at any time point. Similarly, all fluoride products provided significantly superior \%RER versus placebo (all $p<0.0001$ ), whereas no significant difference of this param-
\end{abstract}

\section{KARGER}

E-Mail karger@karger.com www.karger.com/cre

\section{(C) 2016 The Author(s) \\ Published by S. Karger AG, Basel 0008-6568/16/0501-0062\$39.50/0}

This article is licensed under the Creative Commons AttributionNonCommercial-NoDerivatives 4.0 International License (CC BYNC-ND) (http://www.karger.com/Services/OpenAccessLicense) Usage and distribution for commercial purposes as well as any distribution of modified material requires written permission. eter was noted between the fluoride treatment groups. Increasing numerical improvements of \%SMHR and \%RER were observed in all four treatment groups over time $(2,4$, and $8 \mathrm{~h}$ ). The present in situ model is a sensitive tool to investigate intrinsic and fluoride-enhanced rehardening of eroded enamel. All three fluoride toothpastes were more efficacious than placebo, and there were no safety concerns following single dosing in this short-term in situ model.

(c) 2016 The Author(s)

Published by S. Karger AG, Basel

Dental erosion is described as the progressive loss of tooth substance as a result of chemical processes that, unlike those in caries pathology, do not involve bacteria [Layer, 2009; Mason, 2009]. Also unlike caries, which has seen a declining prevalence in western countries, the incidence of dental erosion has been reported to be on the increase [Truin et al., 2004; Chadwick, 2006; Toumba, 2006], and the condition has been highlighted as 'a challenge for the 21st century' [Angmar-Månsson, 2006]. The beneficial effects of fluoride dentifrices for the prevention and management of dental erosion have been evidenced in a large number of in vitro and in situ studies [Bartlett et al., 1994; Attin et al., 1998; Ganss et al., 2001; van Rijkom et al., 2003; Ganss et al., 2004; Hughes et al., 2004; 
Table 1. Number of specimens removed at each posttreatment time point

\begin{tabular}{lrr}
\hline Posttreatment time point & 4-hour subjects & 8-hour subjects \\
\hline $2 \mathrm{~h}$ & & 2 specimens removed \\
$4 \mathrm{~h}$ & N/A specimens removed & 2 specimens removed \\
$8 \mathrm{~h}$ & & \\
\hline \multicolumn{2}{c}{ N/A = Not applicable. } & \\
\hline
\end{tabular}

Fowler et al., 2006; Zero et al., 2006; Schleuter et al., 2007, 2009; Fowler et al., 2009; Hara et al., 2009a; Ganss et al., 2010; Hara et al., 2014], and dentifrice containing $\mathrm{NaF}$ has been demonstrated to remineralize acid-softened enamel and confer protection from further demineralization [Zero et al., 2006; Barlow et al., 2009; Hara et al., 2009b].

A novel gel-to-foam toothpaste has been developed with isopentane $(2 \% \mathrm{w} / \mathrm{w})$ to generate a high volume of microfine foam during brushing. This gel-to-foam technology was designed to enhance toothpaste flavor and organoleptic properties and to encourage adherence to twice-daily brushing. Indeed, gel-to-foam toothpaste has been demonstrated, in an intraoral kinetics investigation, to deliver fluoride and potassium more rapidly to the oral cavity and to be superior at removing oral debris and bacteria versus marketed control toothpastes [Barlow et al., 2008; Hall et al., 2008]. It has been conjectured that actives such as anticavity and antisensitivity ingredients may be more efficiently dispersed to difficult-to-reach areas compared with standard toothpastes. The hypothesis that gel-to-foam toothpastes, by increasing the volume of toothpaste slurry and dispersing it throughout the mouth, may permit increased fluoride deposition on tooth enam$\mathrm{el}$, and consequently enhance protection against erosive insults, has not yet been tested. Conversely, whether such increased intraoral distribution of gel-to-foam toothpaste could reduce fluoride availability on acid-softened enamel surfaces, leading to a reduction of antierosion effects, is unknown.

The present study explored the influence of augmented toothpaste dispersion on fluoride deposition by evaluating the enamel remineralization effects of two gel-to-foam toothpastes and a marketed reference toothpaste, all containing 1,450 ppm fluoride as $\mathrm{NaF}$, in comparison with a placebo toothpaste containing $0 \mathrm{ppm}$ fluoride, using a modified short-term in situ erosionremineralization model [Hara et al., 2009a] in which eroded enamel was treated and allowed to remineralize for 2,4 , and $8 \mathrm{~h}$.

Erosion Remineralization from

Gel-to-Foam Toothpaste in situ

\section{Materials and Methods}

\section{Study Design}

This was a single-center, randomized, blinded (to dental examiner and specimen analyst), placebo-controlled, four-treatment, four-period crossover study using an in situ dental erosion-remineralization model [Zero et al., 2006; Hara et al., 2009a]. During each of the four planned treatment visits, the subjects wore a palatal appliance containing newly prepared bovine enamel specimens. The remineralization effects of the test toothpastes on the specimens were evaluated by calculating the percent surface microhardness recovery (\%SMHR) and the percent relative erosion resistance (\%RER) at 2,4 , and $8 \mathrm{~h}$ during the immediate posttreatment period. Based on the individual preference of each subject to enter either 4- or 8-hour posttreatment evaluation groups, they were then randomly allocated to treatment sequence using a schedule provided by the sponsor. The subjects enrolled in the 4-hour group were evaluated over $4 \mathrm{~h}$ after receiving each of the four treatments, whereas those in the 8 -hour group were evaluated over $8 \mathrm{~h}$ after treatment. A standard in situ erosion-remineralization model [Zero et al., 2006; Hara et al., 2009a] was modified so as to assess study end points at 2, 4, and $8 \mathrm{~h}$ rather than only at $4 \mathrm{~h}$ after treatment. In the standard in situ model eight specimens are removed at $4 \mathrm{~h}$, whereas in the present modification two specimens were removed at $2 \mathrm{~h}$, four at $4 \mathrm{~h}$, and, for relevant subjects, two further specimens at $8 \mathrm{~h}$ following treatment, for exploratory analysis (table 1).

\section{Study Population}

Healthy men and women aged 18-65 years with intact maxillary and mandibular dental arches and unstimulated and stimulated salivary flow rates $\geq 0.2$ and $\geq 0.8 \mathrm{ml} / \mathrm{min}$, respectively, were enrolled. Subjects were excluded if they exhibited current active caries or severe gingivitis or were pregnant or breastfeeding.

This IRB-approved study (IU IRB 1109006882) was conducted in accordance with all laws and local regulations and the Declaration of Helsinki at the Oral Health Research Institute (OHRI), Indiana University School of Dentistry, Indianapolis, Ind., USA. The study was registered at ClinicalTrials.gov (NCT01657903) and the sponsor's website (http://www.gsk-clinicalstudyregister.com/ study/Z6961385). All subjects provided written informed consent to participate prior to screening.

\section{Test Products}

The following test products were evaluated and compared: one low- and one medium-relative dentin abrasivity (RDA) gel-tofoam toothpaste both containing 1,450 ppm fluoride as NaF (Sensodyne ${ }^{\circledR}$ Pronamel $^{\mathrm{TM}}$ Iso-Active ${ }^{\circledR}$ Daily Protection, RDA approx. 
35, and Sensodyne ${ }^{\circledR}$ Pronamel $^{\mathrm{TM}}$ Iso-Active ${ }^{\circledR}$ Gentle Whitening, RDA approx. 100; GSK Consumer Healthcare, Weybridge, UK), a reference, positive-control toothpaste containing 1,450 ppm fluoride as $\mathrm{NaF}$ (Pronamel ${ }^{\mathrm{TM}}$; GSK Consumer Healthcare), and a placebo control toothpaste containing 0 ppm fluoride.

\section{Clinical Procedures}

At the screening visit, eligibility criteria, medical history, current medications, and unstimulated/stimulated salivary flow rates were assessed, and oral soft tissue and oral hard tissue examinations were performed. In addition, each subject had an upper arch impression taken for the purpose of constructing an in situ palatal appliance [Zero et al., 2006; Barlow et al., 2009; Hara et al., 2009a].

Subjects attended a pretreatment in-clinic visit to adjust the palatal appliance fit. Prior to each of four treatment visits, the subjects entered a 2-day washout period during which they used a nonfluoride washout toothpaste twice daily to minimize any carryover effects. On the morning of each treatment visit, the subjects were instructed not to use any toothpaste or fluoride-containing products and not to eat or drink for 30 min before starting treatment.

The subjects wore a palatal appliance holding six (4-hour group) or eight (8-hour group) bovine enamel specimens initially for a 5 -min equilibrium period before brushing the facial surfaces of their natural teeth with the test toothpaste $(1.5 \pm 0.1 \mathrm{~g})$ for $25 \mathrm{~s}$. They then swished the slurry around their mouth for $1 \mathrm{~min}$ so as to allow direct contact with the enamel specimens. All brushing and rinsing procedures were performed under the supervision of the study staff. After expectorating the slurry, the subjects rinsed their mouths with $15 \mathrm{ml}$ of tap water for $10 \mathrm{~s}$ before reexpectorating. Each subject stayed on site and continued to wear the palatal appliance for the remainder of the 4 - or 8 -hour posttreatment period. The participants were requested not to talk for the first $1 \mathrm{~h}$ and not to eat or drink anything with the exception of water for the first $2 \mathrm{~h}$. A 5-min interruption was permitted for all subjects at the 2-hour mark. The participants in the 8-hour group were provided lunch on site at the 4 -hour assessment; before eating, they removed their palatal appliance, rinsed it in deionized water for $10 \mathrm{~s}$, and stored it in a closed denture cup containing moist gauze. After the 30-min lunch break, the participants brushed their teeth with a provided toothbrush and deionized water for $1 \mathrm{~min}$ before reinserting the palatal appliance for a further $4 \mathrm{~h}$, giving a total period in situ lasting $8 \mathrm{~h}$.

\section{Preparation of Palatal Appliance}

Enamel specimens $(5 \times 5 \times 3 \mathrm{~mm})$ were prepared from bovine permanent teeth according to a previously reported method [Zero et al., 1992]. Briefly, blocks were cut from the teeth, polished, sonicated, and rinsed in deionized water. All specimens were checked under a stereomicroscope; those with white spots, cracks, and other defects were rejected.

Lesions were created in the enamel by immersing the specimens in $35 \mathrm{ml}$ of commercially available grapefruit juice ( $\mathrm{pH} 3.30$ \pm 0.1 ) for $25 \mathrm{~min}$ as previously described [Zero et al., 2006; Barlow et al., 2009; Hara et al., 2009a]. A second in vitro challenge was performed following the treatment and remineralization of the specimens in the subjects' mouths, applying the same methodology as used for the creation of the initial lesion [Zero et al., 2006].

\section{Determination of \%SMHR and \%RER}

Surface microhardness was assessed as previously reported [Zero et al., 2006; Hara et al., 2009a]. Briefly, the model analyzes

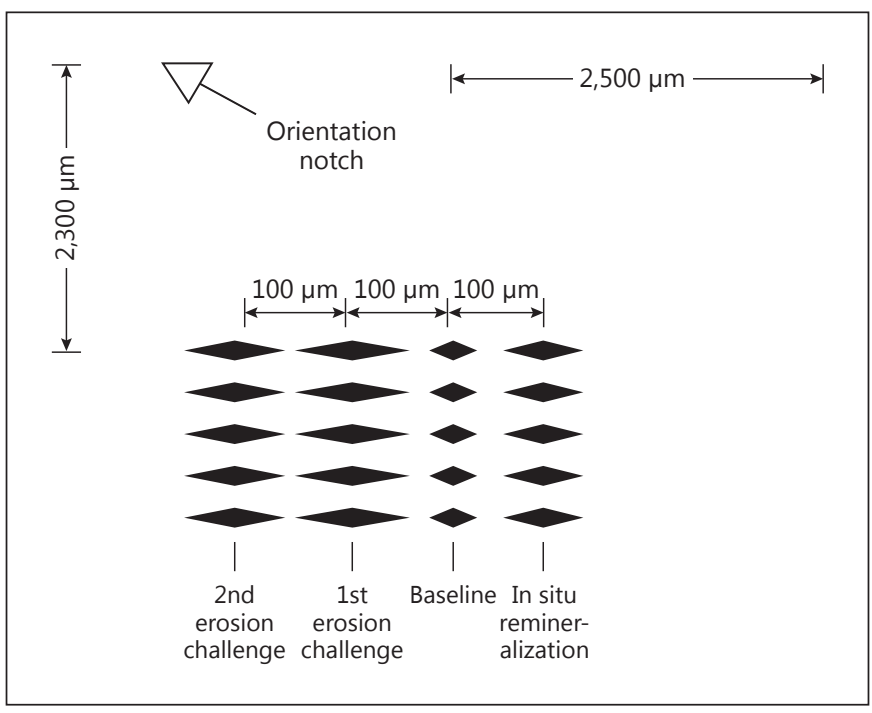

Fig. 1. Sample preparation for in situ model. Freshly prepared bovine enamel specimens were mounted on $25 \times 25$-mm acrylic blocks and a 50-gram load was applied by Knoop diamond for a dwell time of 15 s (2100 HT; Wilson Instruments, Norwood, Mass., USA) so as to create 5 baseline indentations $100 \mu \mathrm{m}$ apart. Indentation lengths were measured following the application of the same load at baseline and after the initial erosive challenge. Enamel specimens were then inserted into a palatal device and worn in situ by subjects during and for 4 or $8 \mathrm{~h}$ following brushing with test toothpastes. Thereafter, the specimens were removed, given 5 postremineralization treatment indentations, reimmersed in acidic drink, and subjected to 5 further indentations (using the same load at all time points) for the investigation of \%SMHR and \%RER at 2, 4, and $8 \mathrm{~h}$ following single treatment applications.

how an equivalent force applied to enamel surface may produce varying indentation lengths (smaller values reflecting greater microhardness and vice versa) under different conditions: at baseline (before treatment; B) and following initial erosive demineralization (E1), remineralization in situ (R), and second extraoral acid challenge after remineralization (E2). Using a Knoop diamond with a load of $50 \mathrm{~g}$ and a dwell time of $15 \mathrm{~s}$ (2100 HT; Wilson Instruments), five baseline indentations were made $100 \mu \mathrm{m}$ apart in the center of the specimen (fig. 1). The indentation lengths were measured and the mean indent length at baseline was derived. The mean indent length was likewise determined after the first erosive challenge, remineralization in situ for 2,4 , or $8 \mathrm{~h}$ (to give \%SMHR at each time point), and the subsequent erosive challenge (to give $\%$ RER after 2, 4, or $8 \mathrm{~h}$ ). Acceptability criteria for specimen inclusion were mean indentation length at baseline of $43 \pm 3 \mu \mathrm{m}$ and an increase of 10-20 $\mu \mathrm{m}$ after the first erosion challenge. The \%SMHR was calculated according to the method of Gelhard et al. [1979] as follows: \%SMHR $=100[(\mathrm{E} 1-\mathrm{R}) /(\mathrm{E} 1-\mathrm{B})]$. As can be seen, the $\%$ SMHR increases as values for $\mathrm{R}$ decrease. Meanwhile, the \%RER was calculated according to the method of Corpron et al. [1986] as follows: $\% \mathrm{RER}=100[(\mathrm{E} 1-\mathrm{E} 2) /(\mathrm{E} 1-\mathrm{B})]$. As the erosion resistance increases, E2 becomes smaller and the \%RER less negative or positive. 


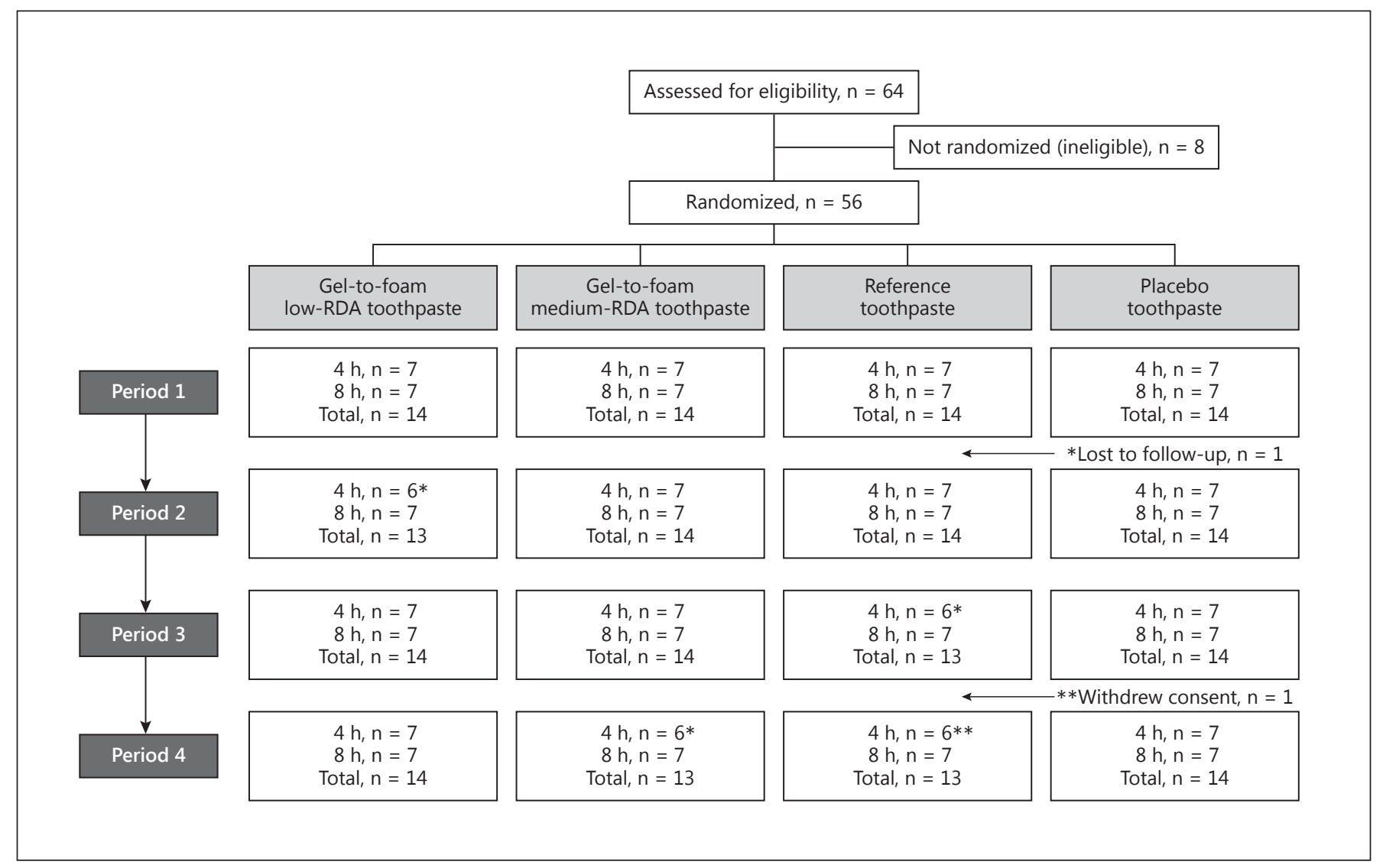

Fig. 2. Allocation of subjects to treatment groups per visit. All subjects were scheduled to receive all 4 treatments; they received 1 of the 4 treatments at each of 4 separate visits in random sequence. Of the 56 subjects randomized, 1 subject was lost to follow-up after completing period 1 and 1 subject withdrew consent after complet- ing period 3. A total of 54 subjects received all 4 randomized treatments and completed the study. Subjects allocated to the 4-hour group provided samples at 2 and $4 \mathrm{~h}$, whereas those in the 8 -hour group provided samples at 2, 4, and $8 \mathrm{~h}$ after treatment.

\section{Statistical Analyses}

The intention-to-treat population was defined as all randomized subjects who had at least one post-baseline efficacy assessment. Efficacy was statistically analyzed in the per protocol population defined as all subjects in the intention-to-treat population who had at least one efficacy assessment considered unaffected by major protocol violations. Safety was assessed in the safety population defined as all subjects who used at least one of the treatment toothpastes during the study.

A sample size of 50 completing subjects was calculated to provide $90 \%$ power to detect treatment differences of $5.4 \%$ in $\%$ SMHR and $9.2 \%$ in $\%$ RER at $4 \mathrm{~h}$ with a $5 \%$ significance level.

For both \%SMHR and \%RER, the test treatments were compared at each time point $(2,4$, and $8 \mathrm{~h}$ ) by analysis of variance (ANOVA) using SAS software, version 9.2 (SAS Institute Inc., Cary, N.C., USA). Models included a random effect for subject and fixed effects for study period, treatment, and planned total intraoral exposure time ( 4 or $8 \mathrm{~h}$ ). All pairwise treatment comparisons for each end point were performed, but only those for gel-to-foam and standard fluoride toothpastes versus placebo were considered

Erosion Remineralization from

Gel-to-Foam Toothpaste in situ primary comparisons. No adjustments for multiplicity were performed because primary comparisons were identified, and all were required to show statistical significance at the 4-hour time point. A p value $<0.05$ was considered statistically significant.

\section{Results}

\section{Study Population}

A total of 64 subjects were screened of whom 56 subjects (male/female, 29/27, 51.8/48.2\%; mean age, $38.7 \pm$ 13.8 years) were randomized between November 2011 and February 2012 (fig. 2). A total of 54 of the 56 randomized subjects (96.4\%) completed the study; 1 subject was lost to follow-up and 1 subject withdrew consent. Twenty-eight subjects each were allocated to the 4- and 8-hour posttreatment evaluation groups. 
Table 2. Between-treatment comparisons: \%SMHR at each time point (per protocol population)

\begin{tabular}{|c|c|c|c|c|}
\hline \multirow[t]{2}{*}{ Time point } & \multirow[t]{2}{*}{ Comparison } & \multicolumn{3}{|l|}{$\%$ SMHR } \\
\hline & & difference & $95 \%$ CI & $\mathrm{p}$ value \\
\hline \multirow[t]{6}{*}{$2 \mathrm{~h}$} & Low-RDA gel-to-foam vs. placebo & 11.1 & $6.9,15.2$ & $<0.0001$ \\
\hline & Medium-RDA gel-to-foam vs. placebo & 11.0 & $6.9,15.2$ & $<0.0001$ \\
\hline & Reference therapy vs. placebo & 10.4 & $6.2,14.6$ & $<0.0001$ \\
\hline & Medium-RDA gel-to-foam vs. low-RDA gel-to-foam & -0.1 & $-4.2,4.1$ & 0.9821 \\
\hline & Reference therapy vs. low-RDA gel-to-foam & -0.7 & $-4.8,3.5$ & 0.7523 \\
\hline & Reference therapy vs. medium-RDA gel-to-foam & -0.6 & $-4.8,3.6$ & 0.7705 \\
\hline \multirow[t]{6}{*}{$4 \mathrm{~h}$} & Low-RDA gel-to-foam vs. placebo & 9.4 & $6.0,12.7$ & $<0.0001$ \\
\hline & Medium-RDA gel-to-foam vs. placebo & 9.5 & $6.1,12.8$ & $<0.0001$ \\
\hline & Reference therapy vs. placebo & 11.7 & $8.3,15.0$ & $<0.0001$ \\
\hline & Medium-RDA gel-to-foam vs. low-RDA gel-to-foam & 0.1 & $-3.3,3.5$ & 0.9607 \\
\hline & Reference therapy vs. low-RDA gel-to-foam & 2.3 & $-1.1,5.7$ & 0.1830 \\
\hline & Reference therapy vs. medium-RDA gel-to-foam & 2.2 & $-1.2,5.6$ & 0.2021 \\
\hline \multirow[t]{6}{*}{$8 \mathrm{~h}$} & Low-RDA gel-to-foam vs. placebo & 8.8 & $3.6,14.1$ & 0.0012 \\
\hline & Medium-RDA gel-to-foam vs. placebo & 11.9 & $6.6,17.1$ & $<0.0001$ \\
\hline & Reference therapy vs. placebo & 13.8 & $8.6,19.1$ & $<0.0001$ \\
\hline & Medium-RDA gel-to-foam vs. low-RDA gel-to-foam & 3.0 & $-2.2,8.3$ & 0.2513 \\
\hline & Reference therapy vs. low-RDA gel-to-foam & 5.0 & $-0.2,10.2$ & 0.0613 \\
\hline & Reference therapy vs. medium-RDA gel-to-foam & 2.0 & $-3.3,7.2$ & 0.4597 \\
\hline
\end{tabular}

Values in italics indicate statistical significance. Difference: difference in adjusted means (first-named treatment minus second-named treatment) such that a positive difference favors the first-named treatment.

Table 3. Between-treatment comparisons: \%RER at each time point (per protocol population)

\begin{tabular}{|c|c|c|c|c|}
\hline \multirow[t]{2}{*}{ Time point } & \multirow[t]{2}{*}{ Comparison } & \multicolumn{3}{|l|}{ \%RER } \\
\hline & & difference & $95 \% \mathrm{CI}$ & $\mathrm{p}$ value \\
\hline \multirow[t]{6}{*}{$2 \mathrm{~h}$} & Low-RDA gel-to-foam vs. placebo & 42.1 & $34.2,50.1$ & $<0.0001$ \\
\hline & Medium-RDA gel-to-foam vs. placebo & 42.6 & $34.6,50.5$ & $<0.0001$ \\
\hline & Reference therapy vs. placebo & 44.0 & $36.0,52.0$ & $<0.0001$ \\
\hline & Medium-RDA gel-to-foam vs. low-RDA gel-to-foam & 0.4 & $-7.6,8.4$ & 0.9154 \\
\hline & Reference therapy vs. low-RDA gel-to-foam & 1.9 & $-6.1,9.9$ & 0.6430 \\
\hline & Reference therapy vs. medium-RDA gel-to-foam & 1.5 & $-6.6,9.5$ & 0.7222 \\
\hline \multirow[t]{6}{*}{$4 \mathrm{~h}$} & Low-RDA gel-to-foam vs. placebo & 41.2 & $34.4,47.9$ & $<0.0001$ \\
\hline & Medium-RDA gel-to-foam vs. placebo & 41.3 & $34.5,48.1$ & $<0.0001$ \\
\hline & Reference therapy vs. placebo & 40.8 & $34.1,47.6$ & $<0.0001$ \\
\hline & Medium-RDA gel-to-foam vs. low-RDA gel-to-foam & 0.1 & $-6.7,6.9$ & 0.9701 \\
\hline & Reference therapy vs. low-RDA gel-to-foam & -0.3 & $-7.1,6.5$ & 0.9257 \\
\hline & Reference therapy vs. medium-RDA gel-to-foam & -0.5 & $-7.3,6.4$ & 0.8965 \\
\hline \multirow[t]{6}{*}{$8 \mathrm{~h}$} & Low-RDA gel-to-foam vs. placebo & 33.3 & $23.9,42.7$ & $<0.0001$ \\
\hline & Medium-RDA gel-to-foam vs. placebo & 28.8 & $19.4,38.2$ & $<0.0001$ \\
\hline & Reference therapy vs. placebo & 32.7 & $23.2,42.1$ & $<0.0001$ \\
\hline & Medium-RDA gel-to-foam vs. low-RDA gel-to-foam & -4.5 & $-13.9,4.9$ & 0.3417 \\
\hline & Reference therapy vs. low-RDA gel-to-foam & -0.6 & $-10.1,8.8$ & 0.8930 \\
\hline & Reference therapy vs. medium-RDA gel-to-foam & 3.9 & $-5.5,13.3$ & 0.4138 \\
\hline
\end{tabular}

Values in italics indicate statistical significance. Difference: difference in adjusted means (first-named treatment minus second-named treatment) such that a positive difference favors the first-named treatment. 


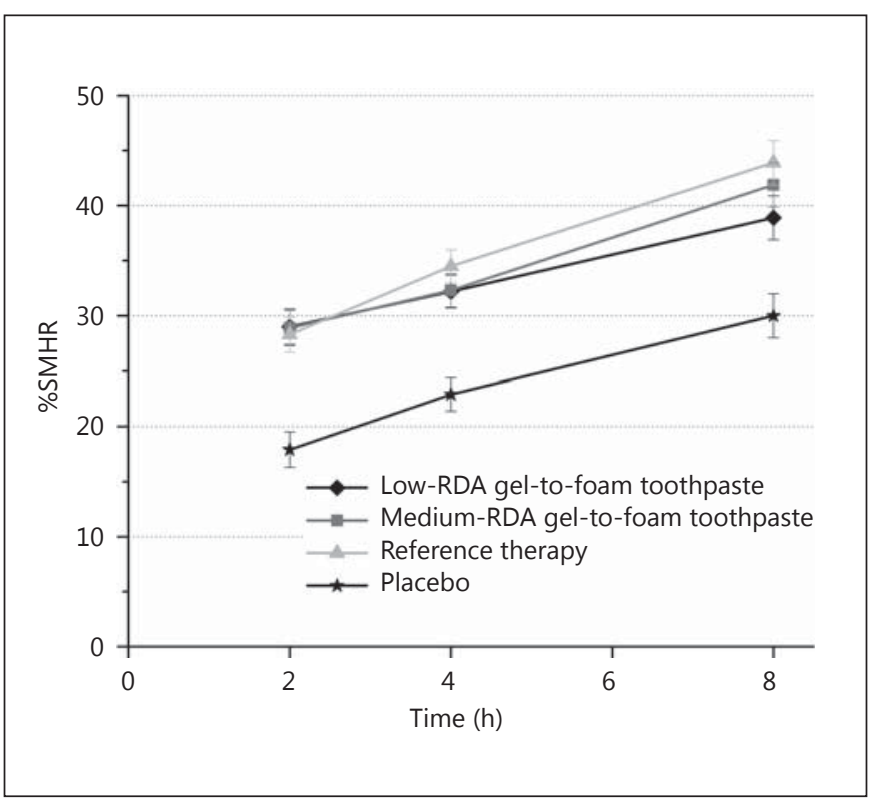

Fig. 3. \%SMHR. Within-treatment unadjusted means and associated between-subject standard errors are displayed. Increases of $\%$ SMHR were observed with longer intraoral exposure for all 4 treatments, with substantially greater increases noted for mediumand low-RDA gel-to-foam toothpastes and reference toothpaste versus placebo.

\section{Efficacy End Points}

All treatments yielded enamel remineralization as evidenced by \%SMHR and \%RER (tables 2, 3; fig. 3, 4). Both low- and medium-abrasivity gel-to-foam fluoride toothpastes and the reference fluoride toothpaste provided significantly greater $\%$ SMHR than placebo at all assessment time points. No statistically significant difference in \%SMHR was observed between the fluoride treatment groups at any time point. Similarly, all fluoride products provided significantly superior \%RER versus placebo, while no significant difference for this parameter was noted between the fluoride treatment groups. Increasing numerical improvements of \%SMHR and $\%$ RER were observed in all four treatment groups over time (fig. 3, 4).

\section{Safety}

A total of 6 adverse events (AEs) were reported during the study, 5 of which were defined as treatment-emergent AEs and 1 washout-emergent AE. Only 1 of the 5 treatment-emergent $\mathrm{AEs}$ was an oral $\mathrm{AE}$ (gingival pain); nonoral AEs included 3 cases of nasopharyngitis and 1 case of back pain. All AEs were mild in intensity, considered

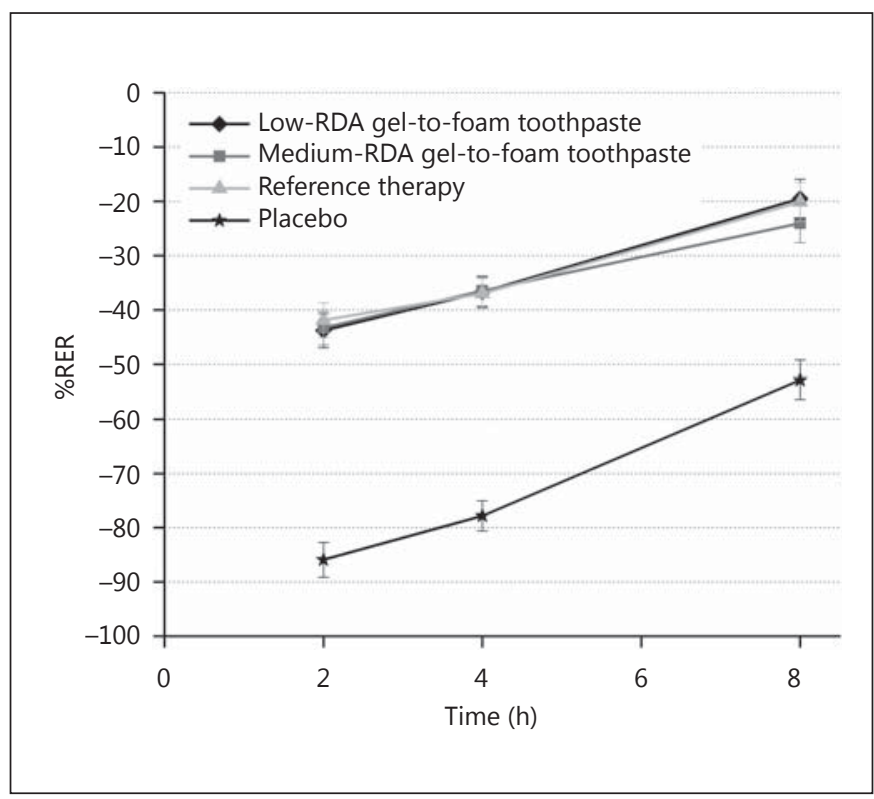

Fig. 4. $\%$ RER at 2, 4, and $8 \mathrm{~h}$. Within-treatment unadjusted means and associated between-subject standard errors are displayed. Increases of \%RER were observed with longer intraoral exposure for all 4 treatments, with substantially greater increases noted for medium- and low-RDA gel-to-foam toothpastes and reference toothpaste versus placebo.

unrelated to the study treatment, and resolved by the end of the study. No serious AEs were reported, and no AE led to study withdrawal.

\section{Discussion}

The present research looked at the effects of enhancing the dispersion characteristics of fluoride toothpastes on their erosion remineralization potential. The experimental gel-to-foam toothpastes contain $2 \% \mathrm{w} / \mathrm{w}$ isopentane which evaporates in the mouth, thereby increasing the volume of foam compared with standard toothpaste, and hence may facilitate intraoral distribution. This study tested whether the two gel-to-foam toothpastes one with low abrasivity and one with medium abrasivity - provided protection from demineralization and effectively remineralized enamel subjected to an erosive challenge using an in situ remineralization model [Zero et al., 2006; Hara et al., 2009a]. The positive control was selected because this toothpaste has been extensively tested in the present in situ model [Zero et al., 2006; Barlow et al., 2009]. A key advantage of using an in situ pal- 
atal appliance model is that specimens can be removed at different time points. Because this study aimed to explore the antierosion efficacy of different novel fluoride formulations, the original in situ model of $4 \mathrm{~h}$ was modified to reveal the extent of remineralization at earlier $(2 \mathrm{~h})$ and later $(8 \mathrm{~h})$ time points, as well as the original 4 -hour time point.

The two gel-to-foam toothpastes and the reference toothpaste elicited statistically greater enamel surface rehardening and erosion resistance versus placebo toothpaste based on \%SMHR and \%RER, respectively, following intraoral exposure at all assessment time points (all $\mathrm{p}<0.0001$ ). However, no statistically significant difference for these efficacy outcomes was observed between the three fluoride toothpastes tested. This finding suggests that increasing the dispersion properties of fluoride toothpastes did not enhance their erosive lesion remineralization efficacy; possibly a higher distribution was offset by a greater dilution of fluoride, leading to no additional net benefit in terms of enamel rehardening. The lack of discernible differences between the medium- and lowabrasivity gel-to-foam fluoride toothpastes and the reference fluoride toothpaste implies that formulating gel-tofoam toothpastes with different concentrations of silica abrasives had no detrimental effects on fluoride's erosion protection of enamel compared with the reference therapy - which has previously demonstrated useful antierosion performance [Zero et al., 2006; Barlow et al., 2009; Hara et al., 2009b].

In the present study, the \%SMHR gradually increased while the \%RER directionally improved in all four treatment groups including placebo over time, and all fluoride treatments were significantly superior to placebo for these parameters at all assessment time points. Some remineralization in the placebo group was not an unexpected result since it is known that saliva promotes the natural deposition of mineral on eroded enamel surfaces [Gedalia et al., 1991; Amaechi and Higham, 2001; Zero and Lussi, 2005]. However, the rehardening of eroded enamel in the placebo group took $8 \mathrm{~h}$ to reach comparable levels to those achieved in the fluoride groups at the 2-hour mark, and remained significantly lower than the fluoride groups at all assessment time points. Further research is required to investigate the remineralization effects of toothpastes at earlier time points $\leq 2 \mathrm{~h}$. The present findings support the concept, at least in principle, that the degree of remineralization increases steadily as fluoride and other minerals/organic materials diffuse from slurry/saliva through the pellicle onto dental hard tissues. These data support the sensitivity of this in situ model and the \%SMHR to detect natural and fluoride-induced rehardening of eroded enamel, which is necessary to preserve tooth structure following acid exposure.

Fluoride-containing toothpastes have been previously confirmed to confer protection against dental erosion caused by acidic foods and drinks using in situ methodology. Barlow et al. [2009] reported a series of studies supporting the effectiveness of fluoride dentifrices to reharden enamel previously softened by erosive challenge and concluded that short-term in situ studies were a sensitive, reproducible, and biologically relevant experimental model. However, the usefulness of fluoride dentifrices in the context of antierosion may be determined by a complex interplay of contributing factors, including not only their free ionizable fluoride content but also fluoride availability and release rate, intraoral mineral ionic configuration (calcium, sodium, phosphate), $\mathrm{pH}$, buffering capacity, toothpaste abrasivity, extent of salivary stimulation, and perhaps above all, compatibility of fluoride with other ingredients [Zero et al., 2006; Lussi et al., 2011]. Indeed, in their set of studies using the present in situ model, Barlow et al. [2009] observed significant differences in the \%SMHR and \%RER exerted by toothpastes matched for fluoride content. Newby et al. [2006] showed that a series of commercial dentifrices isotonic for fluoride concentration differed markedly in their potency to restrengthen and redeposit fluoride in surface-softened enamel in vitro and concluded that enamel fluoride uptake is not simply a function of free fluoride availability - underscoring the importance of formulation effects in driving the antierosive performance of toothpaste. Thus, fluoride toothpaste formulations should be designed and tested so that their active ingredients are released rapidly (within the time frame of toothbrushing) to form durable intraoral reservoirs in contact with tooth surfaces, with low potential for chemical and physical interactions that might interrupt fluoride uptake by dental hard tissues [Shellis et al., 2014].

This study aimed to investigate the impact of including an isopentane foaming agent in a dentifrice formulation on its remineralization potential using a standard in situ erosion model that has been validated in a number of published clinical trials [Zero et al., 2006; Hara et al., 2009a]. In this standard design, all subjects brushed their natural teeth for $25 \mathrm{~s}$ prior to swishing with the toothpaste slurry. If the intraoral fluoride dispersal characteristics of the gel-to-foam dentifrices led to an observable treatment difference in comparison with the reference therapy, further investigation of these products using a different study design would have been devised. 
In conclusion, in this crossover in situ study no statistically significant differences were observed between the two fluoride gel-to-foam toothpastes and an established antierosion fluoride toothpaste (reference therapy) in terms of strengthening enamel, as determined by \%SMHR, and increasing resistance to erosion of previously softened enamel, as surmised by \%RER, at any posttreatment evaluation time point. These findings suggest that the inclusion of $2 \% \mathrm{w} / \mathrm{w}$ isopentane in a fluoridated toothpaste formulation does not inhibit the tooth-hardening action of fluoride, within the limitations of the present study. All three tested fluoride toothpastes were more efficacious than placebo, and there were no safety concerns following single dosing in this short-term in situ model.

\section{Acknowledgments}

The authors thank the subjects who participated and other site staff involved in the conduct of this study, as well as Rita Gala Guibert for clinical operations and Sue Kelly, OHRI, for the overall coordination of the study. We also thank Shalini Dwivedi, Inventiv Health Clinical, and Alex Kahney, Emcee Medical Communications Ltd., for their support in drafting this manuscript. This study was supported by GSK Consumer Healthcare, UK.

\section{Author Contributions}

M.N., P.J., and S.M. contributed to the design and reporting of the study. F.L., A.T.H., and D.T.Z. were involved in the conduct of the study. All authors had access to the final study report, made contributions to the development of the manuscript, had final responsibility for the decision to submit, and approved the submitted version.

\section{Disclosure Statement}

This study was funded by GSK Consumer Healthcare. M.N., P.J., and S.M. are employees of GSK Consumer Healthcare. D.T.Z., A.T.H., and F.L. have received compensation from GSK Consumer Healthcare as independent consultants.

\section{References}

Amaechi BT, Higham SM: Eroded lesion remineralization by saliva as a possible factor in the site-specificity of human dental erosion. Arch Oral Biol 2001;46:697-703.

Angmar-Månsson B: Foreword; in Lussi A (ed): Dental Erosion: From Diagnosis to Therapy. Monogr Oral Sci. Basel, Karger, 2006, pp 1112.

-Attin T, Zirkel C, Hellwig E: Brushing abrasion of eroded dentin after application of sodium fluoride solutions. Caries Res 1998;32:344-350.

Barlow AP, Gross RC, Shanga GM, Barrett K, Baxter K: Removal of oral debris/bacteria by an experimental gel-to-foam toothpaste. Poster presentation (abstract 0467). Pan Eur Fed Int Assoc Dent Res, London, September, 2008.

Barlow AP, Sufi F, Mason SC: Evaluation of different fluoridated dentifrice formulations using an in situ remineralization model. J Clin Dent 2009;20:192-198.

Bartlett DW, Smith BG, Wilson RF: Comparison of the effect of fluoride and non-fluoride toothpaste on tooth wear in vitro and the influence of enamel fluoride concentration and hardness of enamel. Br Dent J 1994;176: 346-348.

Chadwick RG: Dental Erosion. Hanover Park, Quintessence, 2006, p 4.

- Corpron RE, Clark JW, Tsai A, More FG, Merrill DF, Kowalski CJ, Tice TR, Rowe CE: Intraoral effects of a fluoride-releasing device on acidsoftened enamel. J Am Dent Assoc 1986;113: 383-388.
Fowler CE, Gracia L, Edwards MI, Rees GD, Willson R, Brown A: Inhibition of enamel erosion and promotion of lesion rehardening by fluoride: a white light interferometry and microindentation study. J Clin Dent 2009;20:178185.

Fowler CE, Willson RJ, Rees GD: In vitro microhardness studies on a new anti-erosion desensitizing toothpaste. J Clin Dent 2006;17: 100-105.

- Ganss C, Klimek J, Brune V, Schürmann A: Effects of two fluoridation measures on erosion progression in human enamel and dentine in situ. Caries Res 2004;38:561-566.

Ganss C, Klimek J, Schaffer U, Spall T: Effectiveness of two fluoridation measures on erosion progression in human enamel and dentine in vitro. Caries Res 2001;35:325-330.

Ganss C, Lussi A, Sommer N, Klimek J, Schlueter $\mathrm{N}$ : Efficacy of fluoride compounds and stannous chloride as erosion inhibitors in dentine. Caries Res 2010;44:248-252.

Gedalia J, Dakuar A, Shapira L, Lewinstein I, Goultschin J, Rahamim E: Enamel softening with Coca-Cola and rehardening with milk or saliva. Am J Dent 1991;4:120-122.

Gelhard TB, ten Cate JM, Arends J: Rehardening of artificial enamel lesions in vivo. Caries Res 1979;13:80-83.

Hall PJ, Horay CP, Spradbery PS, Hill D, Barlow AP: Intra-oral potassium dispersion profile of a gel to foam toothpaste. Poster presentation (abstract 0466). Pan Eur Fed Int Assoc Dent Res, London, September, 2008.
Hara AT, Barlow AP, Eckert GJ, Zero DT: Novel in-situ longitudinal model for the study of dentifrices on dental erosion-abrasion. Eur J Oral Sci 2014;122:161-167.

-Hara AT, González-Cabezas C, Creeth J, Parmar M, Eckert GJ, Zero DT: Interplay between fluoride and abrasivity of dentifrices on dental erosion-abrasion. J Dent 2009b;37:781-785.

- Hara AT, Kelly SA, González-Cabezas C, Eckert GJ, Barlow AP, Mason SC, Zero DT: Influence of fluoride availability of dentifrices on eroded enamel remineralization in situ. Caries Res 2009a;43:57-63.

Hughes JA, West NX, Addy M: The protective effect of fluoride treatments against enamel erosion in vitro. J Oral Rehab 2004;31:357-363.

Layer TM: Formulation considerations for developing toothpastes suitable for those at risk from erosive tooth wear. J Clin Dent 2009;20: 199-202.

Lussi A, Schlueter N, Rakhmatullina E, Ganss C: Dental erosion - an overview with emphasis on chemical and histopathological aspects. Caries Res 2011;45(suppl 1):2-12.

Mason SC: New in vitro and in situ evidence for a toothpaste formulated for those at risk of acid wear. J Clin Dent 2009;20:175-177.

Newby CS, Creeth JE, Rees GD, Schemehorn BR: Surface microhardness changes, enamel fluoride uptake, and fluoride availability from fluoride toothpastes. J Clin Dent 2006;17:94-99. 
Schleuter N, Ganss C, Mueller U, Klimek J: Effect of titanium tetrafluoride and sodium fluoride on erosion progression in enamel and dentine in vitro. Caries Res 2007;41:141-145.

Schleuter N, Klimek J, Ganss C: Efficacy of an experimental tin-F-containing solution in erosive tissue loss in enamel and dentine in situ. Caries Res 2009;43:415-421.

-Shellis RP, Featherstone JDB, Lussi A: Understanding the chemistry of dental erosion; in Lussi A, Ganss C (eds): Erosive Tooth Wear: From Diagnosis to Therapy. Monogr Oral Sci. Basel, Karger, 2014, pp 163-179.

Toumba J: Tooth surface loss - a challenge to oral health. J Clin Dent 2006;17:85-87.
Truin GJ, van Rijkom HM, Mulder J, van't Hof MA: Dental caries and dental erosion among 5- and 6-year-old and 11- and 12-year-old school children in the Hague, The Netherlands. Changing prevalences? (in Dutch). Ned Tijdschr Tandheelkd 2004;111:74-79.

van Rijkom H, Ruben J, Vieira A, Huysmans MC, Truin GJ, Mulder J: Erosion-inhibiting effect of sodium fluoride and titanium tetrafluoride treatment in vitro. Eur J Oral Sci 2003;111: 253-257.
Zero DT, Fu J, Anne KM, Cassata S, McCormack SM, Gwinner LM: An improved intra-oral enamel demineralization test model for the study of dental caries. J Dent Res 1992;71: 871-878.

Zero DT, Hara AT, Kelly SA, González-Cabezas C, Eckert GJ, Barlow AP, Mason SC: Evaluation of a desensitizing test dentifrice using an in situ erosion remineralization model. J Clin Dent 2006; 17:112-116.

Zero DT, Lussi A: Erosion - chemical and biological factors of importance to the dental practitioner. Int Dent J 2005;55:285-290. 